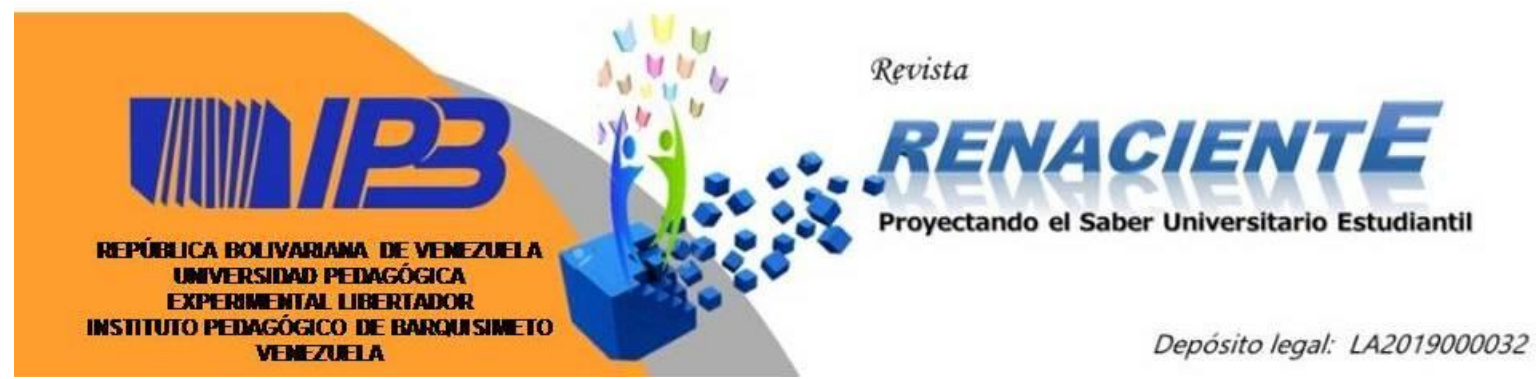

$\begin{gathered}\text { *Andrés A. Cordovés M. } \\ \text { acordoves@ gmail.com }\end{gathered}$
https://orcid.org/0000-0001-7314-8522
**Cristóbal A. de Jesús Liscano
Cristobalbuono@ gmail.com
https://orcid.org/0000-0003-4182-0358
***Marlyn C.Gómez
marlyncarolina13@ @mail.com
https://orcid.org/0000-0003-4187-1833

Estudiantes del Programa de Educación Técnica, Especialidad Educación Comercial

****OOlid Y. Pernalete V. olidpernalete@gmail.com https://orcid.org/0000-0002-1514-001X

Universidad Pedagógica Experimental Libertador Instituto Pedagógico de Barquisimeto (UPEL IPB) Venezuela

\title{
UNA APROXIMACIÓN A LA ESCUELA DE PAZ *****
}

En el presente ensayo se representa un avance de la investigación que estamos realizando en el eje de Práctica profesional III e Investigación II. La misma corresponde a revisar a la escuela como institución social y se analizan los efectos de esta en el proceso educativo venezolano, a la luz del enfoque Escuela de Paz, se fundamenta en las necesidades arrojadas por la Encuesta de la Calidad en la Educación y en los esfuerzos por una articulación curricular que logre la integralidad de la enseñanza, haciendo énfasis en que la dinámica debe surgir de iniciativas propias de un orden social libre en consonancia con los estímulos académicos y las demandas del entorno, orientando el proceso de manera orgánica, espontánea y acorde con los valores y principios del paradigma crítico.

Palabras claves: Escuela de Paz, Articulación Curricular, Proceso Educativo.

\section{AN APROXIMATION TO THE SCHOOL OF PEACE}

* Especialista en comunicación para el liderazgo, escritor, estudiante de Educación Comercial UPEL IPB, cursante del eje Investigación, TIC y Práctica Profesional ** T.S.U. en Mercadotecnia menciones publicidad e investigación de mercado, especialista en comunicación para el liderazgo, estudiante de educación comercial UPEL IPB, cursante del eje Investigación, TIC y Práctica Profesional. *** T.S.U. en Recursos físico y financieros, estudiante de Educación Comercial UPEL IPB cursante del eje Investigación, TIC y Práctica Profesional

****Doctora en Educación, .Docente en los Ejes Investigación, TIC y Práctica Profesional. Miembro activo del Núcleo de Investigación NIDIT de la UPEL-IPB adscrita a la línea TIC y Pedagogía *****El ensayo es un avance de la investigación grupal realizada en el eje de Práctica profesional III e Investigación II del Programa de Educación Técnica.

\section{In}

In this essay it represents advances in the research that we are carrying out in the Professional Practice III and research II axis. It corresponds to reviewing the school as a social institution and its effects on the Venezuelan educational process are analyzed, in light of the School of Peace approach, it is based on the needs thrown up by the Survey of Quality in Education and in the efforts for a curricular articulation that achieves the integrality of the teaching, emphasizing that the dynamics must arise from initiatives of a free social order in line with the academic stimuli and the demands of the environment, guiding the process in an organic, spontaneous way and in accordance with the values and principles of the critical paradigm.

Key words: School of peace, curricular articulation, educational process. 


\section{Introducción}

Con la dinamización social, producto de la masificación de la cultura global, el ser humano, como propulsor y al mismo tiempo recipiente de las constantes actualizaciones de sus modos de vida, en respuesta a su curiosidad innata y a la aceptación de espacios, conocimientos, comportamientos y realidades, ha avanzado en su proceso natural de evolución.

Estos cambios, que no pasan desapercibidos para el docente, como contribuyente en la construcción del individuo social, al despertar sus habilidades investigativas en pro de ofrecer ambientes donde la transición sea mucho más amigable para los nuevos ciudadanos del mundo, hacen natural el surgimiento de visiones que buscan el refrescamiento de las experiencias dentro del ambiente educativo, con las intenciones de cubrir las necesidades emergentes de los nuevos actores sociales.

En algunas oportunidades se manifiestan dentro de la estructura más convencional de los recintos pedagógicos, para ofrecer un merecido refrescamiento del canon establecido, mientras que otras veces se manifiestan de forma irreverente, producto de experiencias contextuales y de las exigencias en la dinámica social, caracterizados en algunos casos por las necesidades de supervivencia e incluso integración de formas no tradicionales de ver el mundo.

Estas formas culturales pueden prefigurar talleres, cursos, asignaturas y carreras, incluso recintos de estudio, escuelas, que pretenden, más allá de cubrir las necesidades o falencias del sistema educativo, fortalecer el proceso de formación, con el objeto de mejorar aquellos valores que surgen naturalmente en la evolución de las sociedades. Este contexto encuentra alivio en la incontrolable marea de cambios que afectan a su población, la mayoría de las veces en detrimento del mismo ser venezolano; lo que la hace un escenario ideal para los espacios ganados a la exploración y la construcción de nuevos valores que se integran a la idea de país, así como en la inserción de nuevos elementos que llevarán al ciudadano a un nuevo estado de consciencia, con una visión fresca y única, dando paso a un hombre nuevo. 
Es allí, en el seno de la transformación social, la innovación y la apropiación de los valores que impulsan el interés educativo, y que representan de forma integral la visón del hombre nuevo, que radica la Escuela de Paz, como un conjunto de espacios ganados para el desarrollo de las múltiples inteligencias, en pro de un ser humano más holístico, a través de la formación complementaria en las más diversas temáticas del saber; y a través de la integración armónica entre los bloques elementales del saber, la tradición educativa, por llamarlos de alguna manera, y aquellos nacientes del querer hacer y querer saber propios de cada individuo en la búsqueda de la libertad de sus saberes.

Al haber encontrado una ubicación estratégica en el seno de la escuela convencional, según la óptica del nuevo modelo curricular venezolano, a través de los grupos estables o Creación, Recreación y Producción (CRP) o de interés, han venido a conformar experiencias para la exploración del ser mismo a través de las artes y la ciencia, no desde el conocimiento digerido y expuesto, como la tradición más clásica lo supone, sino más bien a través de la experiencia contextual, individual de cada uno de sus protagonistas.

También pueden darse en escenarios externos, con características más adecuadas a la exploración de estas rutas artísticas, por llamarlas de alguna manera, por lo que sirven de ejemplo, canchas de entrenamiento, conservatorios de música, laboratorios del pensamiento (ThinkTanks), o escuelas de arte vespertino, que bajo la misma bandera buscan ampliar la perspectiva del venezolano. Es en estos espacios, en los que se genera un enfoque integral, holístico y participativo, con estrategias de instrucción comunes y complementarias, nacidas en su propio seno, de las experiencias más satisfactorias y con la mayor aceptación de todos sus participantes, siendo entonces la Escuela de Paz, más que una estructura física, o un modelo específico, un portafolio de maneras, más allá del tópico de atención, de conexión y de desarrollo del ingenio.

Al buscar, entonces, el desarrollo individual y social de todos sus involucrados, desde el enfoque que parte de la Educación Técnica Comercial, como impulsor de la productividad en el individuo como eje transversal de su desarrollo profesional, se articula en función del encuentro con la creación intelectual como una práctica que favorece el 
emprendimiento y por consiguiente la ganancia del nuevo hombre venezolano, no solo en un aspecto estrictamente moral, sino económico.

Es desde esta intencionalidad que surge el interés del estudio de los grupos estables en Escuelas Técnicas Comerciales y Centros de Formación Alternativa de la ciudad de Barquisimeto, estado Lara, para buscar prácticas didácticas que permitan diseñar estrategias instruccionales bajo el enfoque denominado Escuela de Paz.

Actualmente nos encontramos en el proceso de realizar la metodología a utilizar, pero queremos compartir los primeros avances de lo que consideramos una temática que pudiera ayudar a los grupos estables, interés o Creación, Recreación y Producción (CRP), como quieran definirlo el sistema educativo, a conocer las competencias que cada uno de los estudiantes posee y así valorarse.

\section{Desarrollo Teórico}

\section{De la Escuela, orígenes y desafíos}

La escuela ocupa, en el piélago de instituciones sociales surgidas a partir de la civilización humana, un lugar fundamental como órgano de transmisión y modelaje cultural; su existencia puede rastrearse hasta el neolítico tardío, y como parte de la estructura de las primeras culturas que conformaron Estados, hasta su surgimiento en la antigua Sumeria, regida por una normatividad y sujeta a los intereses de la organización social desplegada por la autoridad para el dominio de sus súbditos.

La escuela que se instaura en América, es la occidental, según Briceño (1977) caracterizada por su evolución ontológica, al ser heredera de la Academia de Platón y del Liceo Aristotélico; pero sus formas no son suficientes para modificar radicalmente la orientación modeladora del individuo, tan solo bastan para hacerla sistemática y universal, es decir, para maximizar sus finalidades modeladoras hasta el punto de despersonalizarla, al convertirla en un aparato burocrático imperial.

Esa escuela colonial, es la que luego de las transformaciones sociales derivadas de las revoluciones liberales, se convertirá en el escenario de numerosos ensayos pedagógicos, dirigidos a la democratización y el acceso de la educación como medio para la 
emancipación individual en aras de formar ciudadanos libres y capaces de aportar a la nación sus potencialidades creadoras para alcanzar óptimos niveles de desarrollo.

La escuela contemporánea, mediada por una singular realidad política en la que intervienen diversos factores, tales como su construcción gnoseológica sobre la base de parámetros científicos y su evaluación y adecuación orgánica a parámetros internacionales, se enfrenta a una sociedad atomizada y polarizada, en la que colisionan y confluyen diversas cosmovisiones y se construyen nuevos paradigmas emergentes, producto de la hibridación cultural y de la globalización socioeconómica, para suponer sobre ella una educación sistémica en términos operativos, sujetos a las contingencias del Estado Docente.

Es sobre esta escuela y sobre esta realidad socioeducativa que se establece un panorama de investigación propicio; habida cuenta de su configuración política, como espacio para el desarrollo de individuos hábiles para el ejercicio de la ciudadanía, su configuración técnica, como contexto de formación para el ejercicio profesional y con esto, la integración productiva del individuo al aparato económico, en calidad de generador de riqueza y su configuración filosófica como escenario para la socialización y la gestación del pensamiento autónomo, reflexivo y crítico, capaz de interpretar las realidades del medio y transformarlas deliberadamente.

La escuela como entidad pública, como institución formal, acreditada por el Estado para la garantía de una educación obligatoria y accesible, según lo establece el artículo 103 y 106 de la Constitución de la República Bolivariana de Venezuela, y la escuela como entidad social, como institución humana, estructurada para cubrir las necesidades e intereses propios de las comunidades que convergen en ella para el desarrollo orgánico, libre y deliberante de la cultura, reflejan en sí mismas dos realidades hasta cierto punto discordantes.

Estas realidades aparentemente incompatibles, la primera forma parte de un sistema teleocrático, tal como lo apunta Hayek, citando a Oakeshott (1966) por estar gobernada por un fin y por constituir entonces un orden social no libre; la segunda se ajusta al concepto de sistema nomocrático, es decir, un orden social libre, solo sujeto al imperativo categórico, según la perspectiva kantiana, en la que la ley no es más que el producto de valores emergentes de una moral universal común a todos los individuos, expresada por el derecho 
negativo; en otras palabras, la restricción de la intromisión tangible o intangible de un sujeto en el espacio de otro.

Desde esta visión se percibe a la educación impartida en la escuela formal como sujeta al currículo prescrito, la actuación docente en este sentido es evaluada y calificada por organismos oficiales de orden jerárquico y el desempeño académico de los estudiantes es individualizado y cuantificable, obedece al establecimiento de una rúbrica que genera un esquema competitivo. Tal y como sostiene Montessori (2003), se traduce en una certificación de competencias para el ingreso en un nuevo nivel de educación; este sistema de escalas tiene como fin la incorporación de los individuos en el aparato productivo nacional; de modo que, el proceso educativo está intervenido por una intencionalidad primaria: la capacitación o formación de las personas para la autosuficiencia.

Mientras tanto, la educación impartida en las escuelas no formales, concebida por La Belle en Pacheco (2007) está sujeta a currículos reales y ocultos diversos que emergen de acuerdo a los saberes y formas didácticas propias de la institución; la actuación docente, en este caso, es validada por las comunidades participantes y valorada de acuerdo a los resultados y el desempeño académico de los estudiantes generalmente es apreciado de acuerdo con las competencias demostradas, configurando un esquema competitivo pero no de escalas, sino limitado a la intencionalidad primaria de este proceso educativo: la autonomía del ser.

La visión común que une a ambas formas educativas es la de producir un individuo autónomo y autosuficiente, es decir, en ambos casos la intencionalidad primaria es la de conducir (docere) a un ser hacia su emancipación, caracterizada por la libertad de acción (autosuficiencia) y la libertad de pensamiento (autonomía); en este sentido la Consulta sobre la Calidad Educativa llevada a cabo por el Ministerio de Educación venezolano en 2014-2015 arrojó resultados sumamente interesantes coincidentes con esta definición de la educación y con la demanda de una enseñanza más holística.

Esta visión oficialista, conlleva a un esfuerzo por parte de las instancias gubernamentales de reforma del currículo para lograr a través de una nueva trama curricular, la integración o al menos la articulación sistémica de las dos escuelas definidas a los fines de este trabajo: la primera como entidad teleocrática y la segunda como entidad 
nomocrática. Todo lo cual se traduce en una visión curricular integradora, en la que el proceso educativo además de tener una naturaleza instrumental, tuviera sentido para la vida, aproximando con esto a ambas realidades y desdibujando hasta donde fuera posible la odiosa línea divisoria entre ambas formas de funcionamiento de la educación venezolana.

Sin embargo, estos esfuerzos oficiales, apenas logrados como ensayos pedagógicos, han estado mediados por diversos procesos sociopolíticos y socioeconómicos que afectan el proceso socioeducativo fundamental que se quiere intervenir; es posible conjeturar, haciendo una revisión no muy exhaustiva de las realidades escolares del país, que la reforma curricular, aunque ha sido asimilada hasta cierto punto por el sistema público de enseñanza, no ha tenido la misma penetración en el resto del sistema educativo, tal como lo señala Rodríguez (2016). Incluso, en algunos escenarios educativos, la escuela privada no solo no ha asimilado las nuevas directrices curriculares, sino que su visión desborda los límites propuestos o consignados en el nuevo diseño curricular prescrito; tal es el caso de la educación católica, por ejemplo, aunque no es el único referente.

Ante este estado de cosas, es imperativo cuestionar la intencionalidad y la capacidad del Estado de producir artificiosamente una articulación unidireccional de ambas realidades educativas. El currículo es todo, ello es esencialmente cierto, pero el currículo oficial, como ha sido constatado por Gimeno (1991) citando a Grundy, está condicionado por las múltiples realidades divergentes y convergentes en el proceso educativo humano; es decir, la escuela oficial, para los fines del Estado, está penetrada por la escuela humana, en la que interactúan los actores y organizaciones infinitas que produce la realidad social incondicionada, no así a la inversa, difícilmente la escuela humana pueda ser absorbida por la acción interventora y planificadora del sistema teleocrático representado por la institucionalidad estatal.

Es entonces, ante la necesidad inobjetable de esta articulación, impostergable para la armonización de la educación venezolana y el logro de sus fines en condiciones de integralidad, que emerge la inquietud de investigar y de encontrar una forma en la que, independientemente de la actuación oficial, se articulen las mejores prácticas tangibles en el sistema formal y las prácticas más favorables halladas en la educación no formal, con la finalidad de diseñar estrategias replicables, sistemáticas, pero no sometidas al esquema 
teleocrático escolar, sino integrantes del sistema nomocrático o socialmente libre de la educación como proceso humano en el enfoque Escuela de Paz.

\section{La Escuela de Paz}

La Escuela de Paz encuentra sustento en el pensamiento de Montessori (2003) respecto de los fines de la educación en contraste con los de la política; mientras la segunda ha de corregir aquellos efectos de su dinámica para evitar la confrontación por las armas, la primera ha de propiciar las condiciones que hagan sostenibles el diálogo, la convivencia y la justicia entre los individuos y sus relaciones, es decir, mientras la política sirve para evitar guerras, la educación asegura la paz de modo perdurable.

De acuerdo con esto se entiende entonces como Escuela de Paz a un enfoque resultante de la síntesis deliberada de prácticas llevadas a cabo dentro de la escuela formal y prácticas ejercitadas en la escuela no formal, que se caractericen por el uso de estrategias que tengan por objeto el centrar la instrucción en el alumno y que consideren la relación maestro-alumno como una relación auténticamente bidireccional, multifactorial, de mutuo conocimiento y aprendizaje en la medida en que den preponderancia a los sentidos, a la creación libre y a la interacción con pares, en un régimen de solidaridad y crecimiento cooperativo.

En este sentido, lo que motiva a indagar la existencia y operatividad de instituciones educativas inscritas en la modalidad de Educación Técnica y de instituciones educativas no formales, en la ciudad de Barquisimeto, de reconocida trayectoria en el ámbito, que ostentan logros por más de una década, haciendo vida útil en comunidades de la ciudad, sirviendo como modelos de interés para incluir el enfoque de Escuela de Paz en la Educación Técnica Comercial en nuestra localidad.

Reconocemos que es una propuesta ambiciosa, pues estamos conscientes de esta realidad que se expone aquí, más que el sentido epistémico, nuestras inquietudes corresponden a los desafíos que se presentan tanto en el plano curricular, como en el plano de la innovación didáctica y también, por qué no, en el plano de la praxis pedagógica. A estas complejidades se unen las dificultades de tipo exploratorio para hallar fuentes y fundamentos teóricos que sustenten la actuación metodológica. Por esta razón, 
consideramos que el paradigma positivista no es suficiente para abarcar la intencionalidad y para satisfacer las demandas del estudio, es menester aligerar el peso cuantitativo desde una perspectiva premeditadamente humanista.

Como todo proceso investigativo posee sus propias dificultades, limitaciones naturales dentro del paradigma en el que se desarrolla la misma(Arias,2003). A ellas han de sumársele las premuras intrínsecas al devenir social, a los precipitados acontecimientos que marcan las agendas políticas y que impactan severamente en la realidad social, afectando no solamente el ámbito inmediato de la cotidianidad, sino también y con mayor fuerza el ámbito de los estudios sociales; la situación del país amerita encontrar puntos de equilibrio entre esas prisas justificadas por los cambios vertiginosos que se producen en el contexto demográfico y sociográfico nacional y las necesarias prospectivas científicas que permitirán la concentración en la direccionalidad de las actividades experimentales.

Los aspectos mencionados en los párrafos anteriores, consideramos que son claves en la concepción de la Escuela de Paz, para la construcción de una sociedad capaz de asumir desafíos tan relevantes como su incorporación a la sociedad del conocimiento, la asimilación de una actitud determinada a la superación de escollos que dificultan su asunción al progreso y el medio ambiente idóneo para la creación intelectual productiva, para la concepción de una vida con base en la ética y para la transformación interior auspiciada por el encuentro de una dimensión axiológica nutrida desde una mirada filosófica y una profunda sensibilidad artística.

Desde esta perspectiva, los grupos de Creación, Recreación y Producción (CRP), también conocidos como grupos de interés o grupos estables, permiten una aproximación más sincera con los estudiantes de las Escuelas Técnicas para incorporar el enfoque de Escuelas de Paz y configurar una modalidad que permite entender al ser humano en su contexto laboral. Estos CRP son un escenario ventajoso para articular las prácticas didácticas originales y en sumo grado integradoras de saberes con prácticas de la escuela no formal, sobre todo porque en dichos grupos ha de priorizarse la sensibilidad y reflexividad de los participantes. Es posible que se encuentren en ellos numerosas estrategias que se desprendan con mayor facilidad de aquellas provistas en el tejido programático oficial. 
No es que el currículo oficial sea una amenaza o un enemigo de la educación, es que su naturaleza limitativa, coactiva, sujeta a la evaluación cuantitativa y al despliegue de actividades para lograr fines prefijados y adheridos a una voluntad aglutinante, como lo sustenta Gimeno (1991) pueden entorpecer; sino más bien distorsionar la consecución de aquello inmanente en el proceso educativo, del hecho humano ulterior, genésico del que brotan los potenciales creadores del docente como individuo vinculado emocional, cognitiva y vocacionalmente a los estudiantes.

Esta es la razón fundamental por la que el interés primario de nosotros como noveles investigadores es el currículo real y el oculto en las prácticas de los grupos estables como ambientes libres dentro de la escolaridad formal, además de que es la manera más eficaz de contrastar y sintetizar dichas prácticas con las que puedan aparecer en las observaciones dentro de la escuela no formal.

Reforzado esto en Rodríguez (2016) quien aborda la complejidad de los grupos estables invitando a "pensarlos como oportunidades para practicar las competencias del siglo XXI, para el conocimiento y producción de tecnologías con vistas a la producción de bienes y servicios pertinentes a nuestra sociedad.” (p.6). todo ello en virtud que las competencias del siglo XXI exigen primero que nada, una noción distinta de conocimiento.

El conocimiento para los autores Reche y González (2010), es el ejercicio activo, voluntario, de la razón como medio para el procesamiento de datos para la disposición de información que sirva como instrumento para la conexión y transformación del entorno. El conocimiento entonces no es solo una cualidad fisiológica exclusivamente atinente a los procesos básicos y complejos del pensamiento, es una actitud y como tal integra elementos de tipo cognitivo, axiológico, afectivo y conductual, en este sentido el conocimiento visto a la luz del siglo XXI equivale a la cosmovisión y actuación coherente del individuo con el saber, el deber ser, el querer o desear y el hacer.

El enfoque Escuela de Paz se sustenta sobre esta definición de conocimiento, su intencionalidad de incorporar, en la articulación de las prácticas educativas formales y no formales "inventariables", un elemento capital que garantiza además la formación integral del ser en todas sus dimensiones, el desarrollo de las inteligencias múltiples como aspectos cruciales en la develación vocacional, en la construcción de destrezas y en el 
descubrimiento de talentos que lejos de servir para la diferenciación de los individuos, sirvan para el autoconocimiento y en estos términos para la explicitación y afirmación de la identidad individual.

Acentúese que la Escuela de Paz procura una educación liberal, en un sistema nomocrático, como alternativa para la formación complementaria, destacándose en ella la relación docente-estudiante en un contexto igualitario, conducente con el paradigma crítico en el que ambos individuos son concebidos como partes de un proceso de investigación (Habermas,1972), ambos son descubridores y como tales poseen suficiencia comunicacional por lo que el hecho educativo se verá exteriorizado a partir de una actitud cognoscente.

Hasta el momento se ha hecho todavía muy poco para considerar que todo puede decirse o que lo dicho es absoluto; lo revelado por los autores da cuenta de sus someros avances que principian un trabajo meticuloso, escrupuloso y cauteloso; los medios metodológicos para lograr la recopilación de datos y para procesar la información y sistematizarla, también pueden verse como medios ambiciosos, de alguna manera soportan con originalidad la intencionalidad de nuestro estudio.

\section{Reflexiones finales}

La Escuela de Paz como enfoque supone un reto, asumido en condiciones quizás no del todo deseables para los docentes pertenecientes al sistema educativo actual y, más aún, para los docentes en formación. Pero debemos formarnos en el área de la investigación que permite abrir horizontes de soluciones a nuestro contexto. Reto tomado para proyectar nuestra vocación científica y pedagógica en un contexto particularmente diferenciado del destinado para la mayoría de los profesores egresados de la Universidad Pedagógica Experimental Libertador (UPEL). La misma da cuenta de su mirada al mundo y de su voluntad de cambio, no desde la retórica actuante en la inercia del sistema teleocrático, productor de formadores sistemáticos, empleados en las distintas instancias de la formalidad escolar, sino desde la argumentación filosófica y el compromiso paradigmático de profesores con una visión liberal de la educación en condiciones libres de pleno significado para ejercer la virtud docente de manera trascendente. 
Esto es posible, ya que el Diseño Curricular 2015 hace aún más pertinente la dimensión semántica de esta postura profesional; la universidad como formadora de ciudadanos íntegramente comprometidos con la educación como medio para la superación personal, brinda oportunidades para el encuentro significativo con las realidades del sistema y su valoración intersubjetiva. El paradigma crítico sobre el que se forman los estudiantes upelistas a partir del año 2018 es fuente de vital importancia para la asunción y afirmación de la autonomía docente y de la responsabilidad individual en el proceso educativo.

Los educadores no solo están dentro del sistema formal, la educación es un proceso humano, que va más allá de la organización sistemática, es un orden social libre que existe por la libertad y para la libertad, que configura numerosas organizaciones, que no está hecho de asimilación y repetición, sino de auténtico conocimiento en ejercicio práctico y para la vida; la convivencia social, la paz, solo será posible cuando la educación venezolana sea asumida como plano social independiente de los fines del Estado.

Es por ello que consideramos investigar sobre el enfoque de Escuela de Paz, para buscar prácticas didácticas que permita diseñar estrategias instruccionales, a través de la articulación de los grupos estables-CRP o grupo de interés- en las Escuelas Técnicas Comerciales en conjunto con los Centros de Formación Alternativa de la ciudad de Barquisimeto; y así aminorar las necesidades arrojadas por la Encuesta de la Calidad en la Educación y asumir los esfuerzos por una articulación curricular que logre la integralidad de la enseñanza, haciendo énfasis en que la dinámica debe surgir de iniciativas propias de un orden social libre en consonancia con los estímulos académicos y las demandas del entorno, orientando el proceso de manera orgánica, espontánea y acorde con los valores y principios del paradigma crítico.

\section{Referencias}

Arias, F. (2006).Mitos y errores en la elaboración de tesis y proyectos de investigación. Editorial Episteme.

Briceño, José Manuel. (1994). El Laberinto de los Tres Minotauros. Monte Ávila Editores Latinoamericana. 
Gimeno, J. (1991). El curriculum: una reflexión sobre la práctica. http://convivenciajt.weebly.com/uploads/2/6/7/3/26732425/gimeno_1991_cap_1_ aproximacin_al_concepto_de_currculum_fragmento.pdf.

Habermas, J. (2012) Teoría de la acción comunicativa. Acción y racionalidad comunicativa en el marco de la teoría crítica. http://revistas.pucp.edu.pe/index.php/estudiosdefilosofia/article/view/503

Hayek, F. (1966). Principios de un orden social liberal. Trabajo presentado en el encuentro de Tokio de la $\quad$ Sociedad http://institutoamagi.org/download/HayekFriedric-

Losprincipiosdeunordensocialliberal.pdf.

Montessori, M. (2003). El método de la pedagogía científica. https://es.scribd.com/document/355746974/2-El-Metodo-de-la-Pedagogiacientifica-Montessori-pdf.

Pacheco, M. (2007) Educación no formal, concepto básico en educación ambiental. https://comenio.files.wordpress.com/2007/08/noformal.pdf

Reche y González (2010), Las demandas formativas del alumnado de magisterio. Construcción de un plan de formación complementaria. Revista Javeriana. 2 (4) htts://doi.org/10.11144/Javeriana.m2-4.dfam

Rodríguez, N. 2016. Reflexiones sobre el cambio curricular en educación media. http://www.cerpe.org.ve/tl_files/Cerpe/contenido/documentos/Actualidad\%20Educa tiva/Transformacion\%20curricular\%20EM/Reflexiones\%20sobre\%20el\%20cambio $\% 20$ curricular\%20en\%20educacion\%20media.pdf 